\title{
Efficacy of percutaneous transforaminal endoscopic decompression treatment for degenerative lumbar spondylolisthesis with spinal stenosis in elderly patients
}

\author{
XIN-FENG LI ${ }^{1,2^{*}}$, LIN-YU JIN ${ }^{2 *}$, ZHEN-DONG LV ${ }^{2}$, XIN-JIN SU ${ }^{2}$, \\ KUN WANG $^{2}$, HONG-XING SHEN ${ }^{2}$ and XIAO-XING SONG ${ }^{3}$
}

\begin{abstract}
${ }^{1}$ Department of Orthopaedic Surgery, Baoshan Branch of Renji Hospital, School of Medicine, Shanghai Jiaotong University, Shanghai 200444; ${ }^{2}$ Department of Orthopaedic Surgery, Renji Hospital, Shanghai Jiaotong University School of Medicine, Shanghai 200127; ${ }^{3}$ Department of Anesthesiology, Ruijin Hospital, Shanghai Jiaotong

University School of Medicine, Shanghai 200025, P.R. China
\end{abstract}

Received June 17, 2019; Accepted November 13, 2019

DOI: $10.3892 / \mathrm{etm} .2019 .8337$

\begin{abstract}
The efficacy of fusion combined with decompression for the treatment of spinal stenosis with degenerative lumbar spondylolisthesis (DLS) has been debated. Percutaneous transforaminal endoscopic decompression (PTED) under local anesthesia is an ultra-minimally invasive procedure. The present study aimed to evaluate whether PTED is an effective alternative therapy for spinal stenosis associated with DLS in elderly patients. PTED was performed in elderly patients exhibiting lumbar stenosis and low-grade (Meyerding grades I and II) DLS; these patients also exhibited leg-dominant symptoms and had tolerable or absent mechanical back pain. Administration of general anesthesia may be considerably hazardous in patients when combined with comorbid conditions that result from aging. Therefore, the present procedure was performed under local anesthesia. No obvious radiographic lumbar intervertebral instability was identified prior to surgery. Pre- and post-operative visual analogue scale (VAS) score, Oswestry Disability Index (ODI) and walking distance data were collected. The clinical global outcomes following surgery were evaluated using modified MacNab
\end{abstract}

Correspondence to: Dr Xin-Feng Li, Department of Orthopaedic Surgery, Baoshan Branch of Renji Hospital, School of Medicine, Shanghai Jiaotong University, 1058 Huan Zheng Bei Road, Shanghai 200444, P.R. China

E-mail: 1xfrenji@126.com

Dr Xiao-Xing Song, Department of Anesthesiology, Ruijin Hospital, Shanghai Jiaotong University School of Medicine, 197 Ruijin Er Lu, Shanghai 200025, P.R. China

E-mail: littlestar77@126.com

${ }^{*}$ Contributed equally

Key words: degenerative lumbar spondylolisthesis, spinal stenosis, percutaneous transforaminal endoscopy, decompression criteria. A total of 18 elderly patients underwent surgery using PTED techniques. The mean follow-up time was 27.7 months (range, 24-33 months) and the mean estimated blood loss was $18.33 \mathrm{ml}$ (range, 10-35 ml). The mean pre-operative ODI, VAS score of the back and VAS score of the leg were 68.2 \pm 6.5 , $2.8 \pm 1.4$ and $6.6 \pm 1.2$, respectively. All average scores improved post-operatively to $31.7 \pm 5.2,1.5 \pm 0.6$ and $1.7 \pm 0.8$, respectively, at the latest follow-up. A statistically significant improvement was observed for all scores at 1 month and that the scores remained relatively stable after that. According to modified MacNab criteria, the good-to-excellent rate was $83.3 \%$. Only 1 patient required micro-decompression surgery due to poor rating. The present study indicated that PTED may be an effective alternative therapeutic option for elderly patients with low-grade DLS associated with spinal stenosis. However, PTED techniques continue to evolve and further follow-up studies are required to determine the long-term outcomes of this treatment technique.

\section{Introduction}

With the aging of the population, spinal stenosis with degenerative lumbar spondylolisthesis (DLS) in elderly patients has become an increasingly common condition (1). Neurogenic claudication and radiculopathy are the most common symptoms of spinal stenosis (2). Surgery may be offered to patients who are symptomatic and fail to respond to non-operative treatment measures, including physical therapy and epidural steroid injections. Patients are frequently treated using a decompression operation of neural structures. However, the management of these patients remains controversial (1). Several surgical methods have been used for the management of DLS, including decompression without fusion, posterolateral in situ fusion, posterolateral instrumented fusion with pedicle screws, anterior lumbar interbody fusion, posterolateral instrumented fusion with pedicle screws plus interbody fusion and dynamic stabilization (3). In recent years, various studies have assessed the efficacy of decompression alone vs. decompression with fusion for this condition, but the results were conflicting (3-7). 
The focus of controversy was whether the addition of fusion added benefit to decompression for patients with either grade I or II spondylolisthesis spondylolisthesis. Increasing evidence has demonstrated that in the management of DLS associated with spinal stenosis, additional fusion may not yield any clinical improvement over treatment with decompression alone (5-7).

Minimally invasive decompression, which involves a small incision, causes significantly less tissue disruption than open surgery and provides greater patient satisfaction, and is increasingly and more frequently used for DLS (8). Certain studies have indicated that microendoscopic laminotomy is also an effective procedure for the treatment of patients with DLS $(8,9)$. Minimally invasive decompression may prevent post-operative instability and lead to the preservation of stabilizing structures, avoiding the requirement for fusion. Therefore, minimally invasive decompression procedures have been attracting attention in cases of radiating pain-dominant DLS that is associated with spinal stenosis without severe segmental instability (9).

In recent years, percutaneous endoscopic discectomy has been confirmed as an ultra-minimally invasive option for the treatment of lumbar herniated discs (10). Compared with microendoscopic discectomy, percutaneous transforaminal endoscopic discectomy may lead to an increased recovery time and result in an improved clinical outcome (11). Furthermore, a number of studies have demonstrated that percutaneous endoscopic decompression under local anesthesia may also be an efficient alternative to conventional open lumbar decompression surgery for the treatment of lumbar stenosis in elderly patients, while administration of general anesthesia may potentially be a considerable hazard $(12,13)$. In the present study, it was hypothesized that the percutaneous transforaminal endoscopic decompression (PTED) procedure may be effective in cases of radicular pain-dominant DLS, which is associated with spinal stenosis, without any obvious segmental instability. The purpose of the present study was to determine the efficacy of PTED by evaluating the clinical outcome of a group of patients with DLS.

\section{Materials and methods}

Patients. The present investigation was a retrospective cohort study, which was approved by the Ethics Committee of Renji Hospital (Shanghai, China) and aimed to evaluate the outcome of PTED treatment in elderly patients with lumbar spinal stenosis associated with DLS. A total of 750 patients with spinal diseases were treated at the Department of Spine Surgery of Renji Hospital (Shanghai, China) between November 2015 and November 2016; 56 patients with DLS were identified and 18 patients with DLS and spinal stenosis aged $>65$ years, with comorbidities, were included. X-ray was used to diagnose DLS and CT and MRI were used to indicate the compression position. Radiographic instability was determined using flexion-extension radiographs. Patients who had previous lumbar surgeries, trauma, tumors or infection were excluded. The demographic characteristics, radiographic and clinical outcomes and surgery were recorded, and the following inclusion and exclusion criteria were selected with reference to a previous study (14): i) Low-grade (Meyerding grades I and II) DLS; ii) no obvious radiographic lumbar intervertebral instability; iii) symptoms of unilateral radicular leg pain with no or mild back pain; iv) failure of conservative therapies at $>3$ months; and v) elderly patients with potentially considerable hazards for general anesthesia due to severe comorbidities, including heart failure, diabetes mellitus, coronary insufficiency and respiratory failure. Patients enrolled in the present study were excluded according to the following criteria: i) Lumbar spine pathologic conditions, including trauma, tumor or infection; ii) significant facet effusions indicated during MRI (determined as the largest distance between the apparent articular surfaces); and iii) a history of lumbar surgery. A total of 18 patients were successfully included in the current study (average age, 71.2 years; age range, 66-85 years; 10 females and 8 males).

Surgical technique. All patients underwent PTED procedures. All operations were completed by one surgeon with $>5$ years of surgical experience. The procedures were performed using a PTED system (TESSYS ${ }^{\circledR}$; Joimax Co.). Patients were placed in the prone position on a radiolucent table for standard anteroposterior and lateral radiographs to be obtained during intra-operative fluoroscopy. The level of the responsible segment was subsequently determined using $\mathrm{C}$-arm fluoroscopy. The skin entry point was superior to the iliac crest and 9-13 cm lateral from the midline, depending on the patient's waist size. Following surface anesthesia with $1 \%$ lidocaine, needle entry tract anesthesia was performed using a 18-gauge spinal needle with 8-10 ml 1\% lidocaine. The facet joint was anaesthetized using $1 \%$ lidocaine when the needle was engaged with the superior articular processes, and a guide wire was placed into the needle. A 7-mm cut was created via the entry point of the guide wire. The sequential dilators were used to establish a muscle gap approach and bone drills were used to enlarge the foraminal area by removing bone from the anterior lateral portion of the upper articular process. Finally, the working cannula was placed along the guide wire (Fig. 1). The endoscopic system combined with a $0.9 \%$ saline rinsing bag was placed into the working cannula. Nerve decompression was then performed using this system. The hypertrophic ligament flavum, perineural scar and extruded disc material were removed with different instruments, including a radiofrequency knife, punch forceps and nucleus forceps. In order to obtain adequate nerve decompression, foraminoplasty was performed using an endoscopic chisel (Fig. 2A and B) or endoscopic trephine (Fig. 2C and D) to remove hypertrophied superior articular processes. Completely decompressed nerve roots were easily moved and pulsations were indicated to be consistent with the heart rate. Bleeding was stopped by squeezing the rinsing bag or using a bipolar coagulator. After adequate hemostasis, the endoscopic system was removed and the incision was sutured. At $2 \mathrm{~h}$ after surgery, patients were allowed to move when no complications occurred.

Outcome assessment. Imaging methods, including X-ray, CT and MRI, were used to determine radiological outcomes. The improvement in walking distance prior to and after the operation was rated using three different degrees (worse, no change or better). The average visual analogue scale (VAS) score (15), Oswestry Disability Index (ODI) (16) and modified MacNab criteria (17) were determined as the clinical outcomes. The 
A

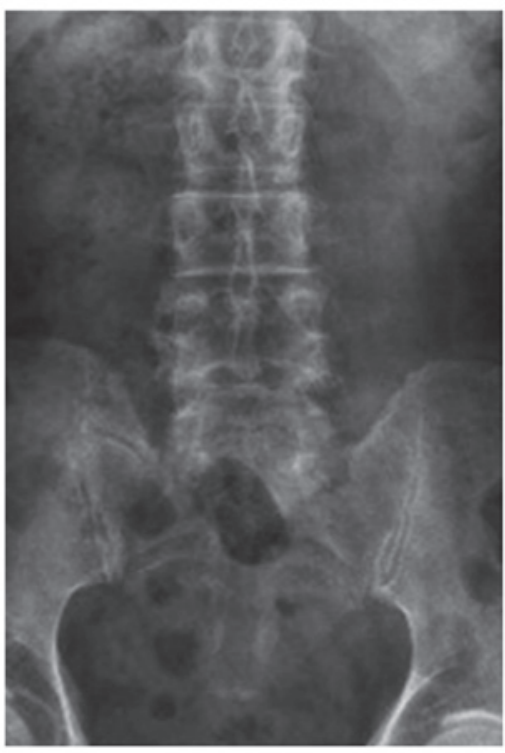

C

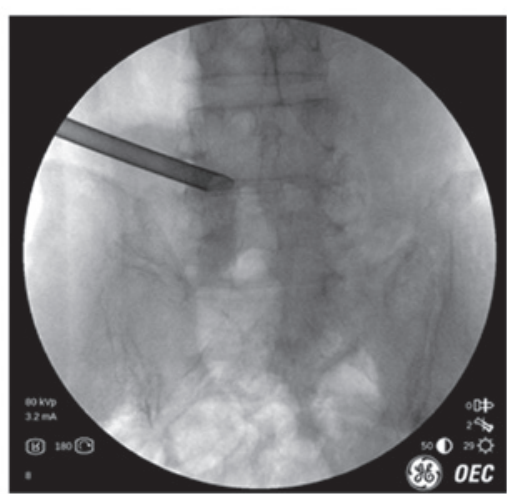

B

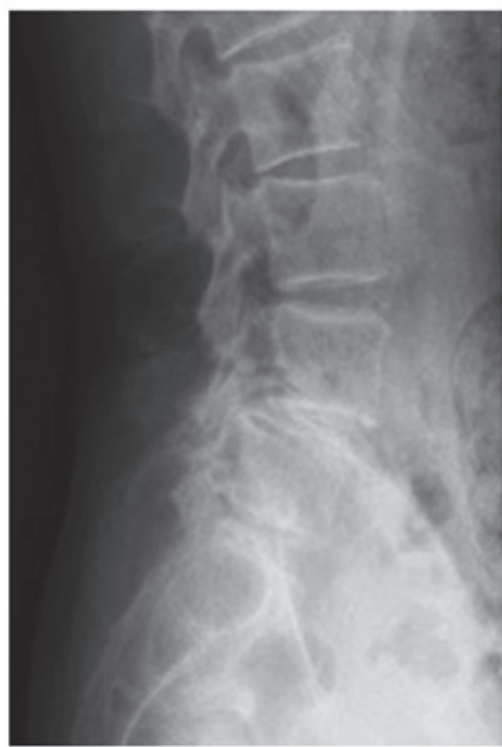

D

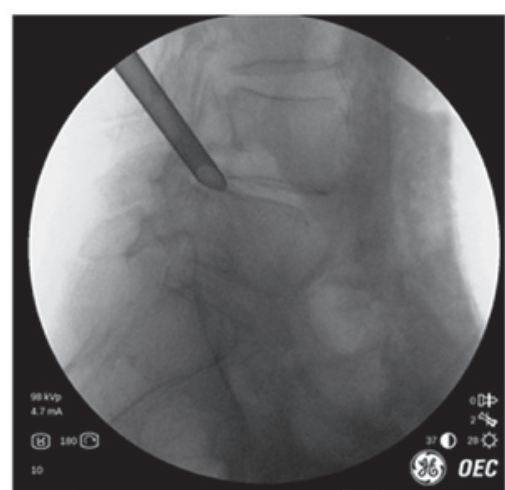

Figure 1. Intra-operative fluoroscopic images of the location of the working cannula in a 79-year-old male patient. Pre-operative (A) anteroposterior and (B) sagittal X-ray images implied a Meyerding grade I DLS. The (C) anteroposterior and (D) sagittal view of the location of the working cannula, using fluoroscopy, is illustrated prior to endoscopic manipulation. DLS, degenerative lumbar spondylolisthesis.

data were obtained using patient questionnaires. All variables were collected at 1 and 3 months following surgery and at a mean follow-up of 27.7 months. Nerve decompression was demonstrated on radiographic imaging examination. For all patients, the percentage slip was measured from the lateral radiographs of the lumbar spine. The sliding distance was determined as the distance between the trailing edge of the vertebral body, below the sliding vertebra, and the parallel line extending through the posterior border of the vertebra, and this was defined as $b$. The percentage slip was defined as the ratio of $b$ to the front and back dimensions of the sliding vertebral body (a): $\%$ of slip=(b/a) x100\%.

Statistical analysis. Statistical analysis was performed using one-way analysis of variance or a paired t-test with SPSS 19.0 (IBM Corp.) to compare the differences in the mean of the outcome scores prior to and after surgery. The Bonferroni test was used for post-hoc comparison. $\mathrm{P}<0.05$ was considered to indicate statistical significance.

\section{Results}

Demographic characteristics and outcomes. Demographic characteristics of the patients and some peri-operative and post-surgical parameters are listed in Table I. The mean body mass index was $24.1 \pm 3.0 \mathrm{~kg} / \mathrm{m}^{2}$. The prevalence of comorbidities among the patients was as follows: Hypertension (61.1\%); diabetes mellitus (27.8\%); heart disease (38.9\%); cerebrovascular infarction (11.1\%); respiratory diseases (16.7\%); renal/ureteral disease (16.7\%); and peripheral vascular disease $(11.1 \%)$.

Clinical outcomes. Modified MacNab criteria were applied in the present study to evaluate the outcomes (Table II). The good-to-excellent rate was $83.3 \%$. A total of 2 patients rated their outcome as fair and 1 patient as poor until three months of the follow-up and this patient underwent subsequent micro-decompression surgery for correction.

The mean pre-operative ODI was $68.2 \pm 6.5$ and the VAS pain score of the leg and back were $6.6 \pm 1.2$ and $2.8 \pm 1.4$, respectively (Fig. 3). Improved outcomes were reported by patients post-operatively $(32.3 \pm 5.6,2.2 \pm 0.9,1.5 \pm 0.7$ at 1 month; $29.9 \pm 5.0,1.8 \pm 1.1,1.3 \pm 0.7$ at 3 months; and $31.7 \pm 5.2$, $1.7 \pm 0.8,1.5 \pm 0.6$ at the latest follow-up (Fig. 3). A significant improvement was observed at 1 month, 3 months and at the latest follow-up in the ODI and VAS score for the leg $(\mathrm{P}<0.01)$, and in the VAS score for the back $(\mathrm{P}<0.05$; Fig. 3$)$. The patients' estimated walking distance increased and an improvement 
A
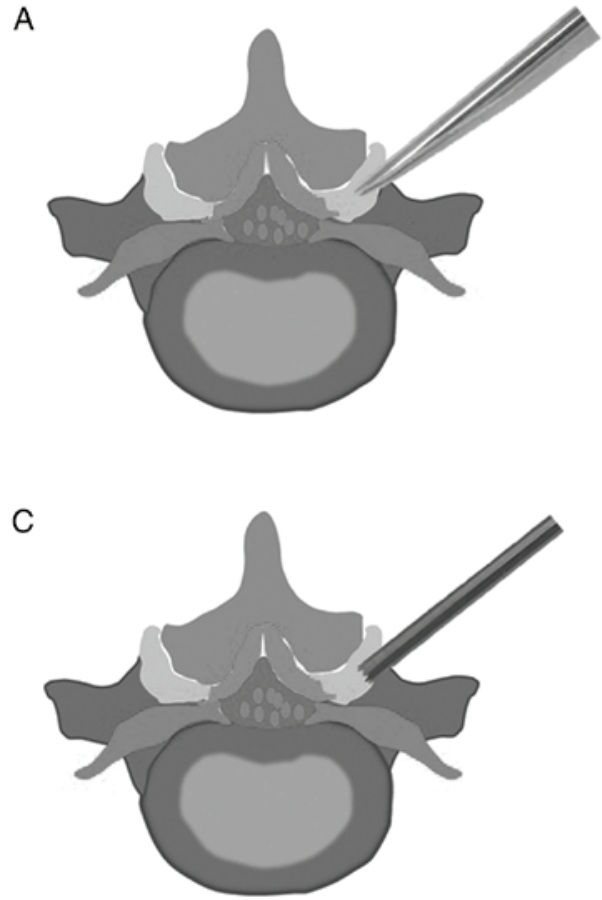

B

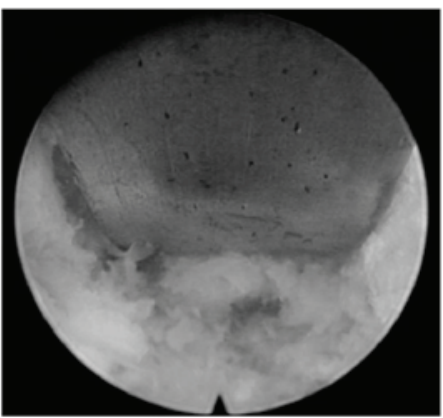

D

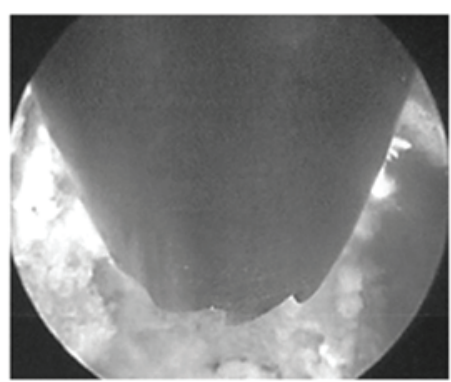

Figure 2. An endoscopic chisel and trephine was used to remove the hypertrophied superior articular processes for foraminal decompression. (A) Illustration of the endoscopic chisel decompression procedure. (B) Intraoperative image indicating endoscopic decompression using chisel at the superior articulating process to expand the foraminotomy in a 67-year-old female patient. (C) Illustration of the endoscopic trephine decompression procedure. (D) Intraoperative image of endoscopic trephine decompression in a 78 -year-old female patient.
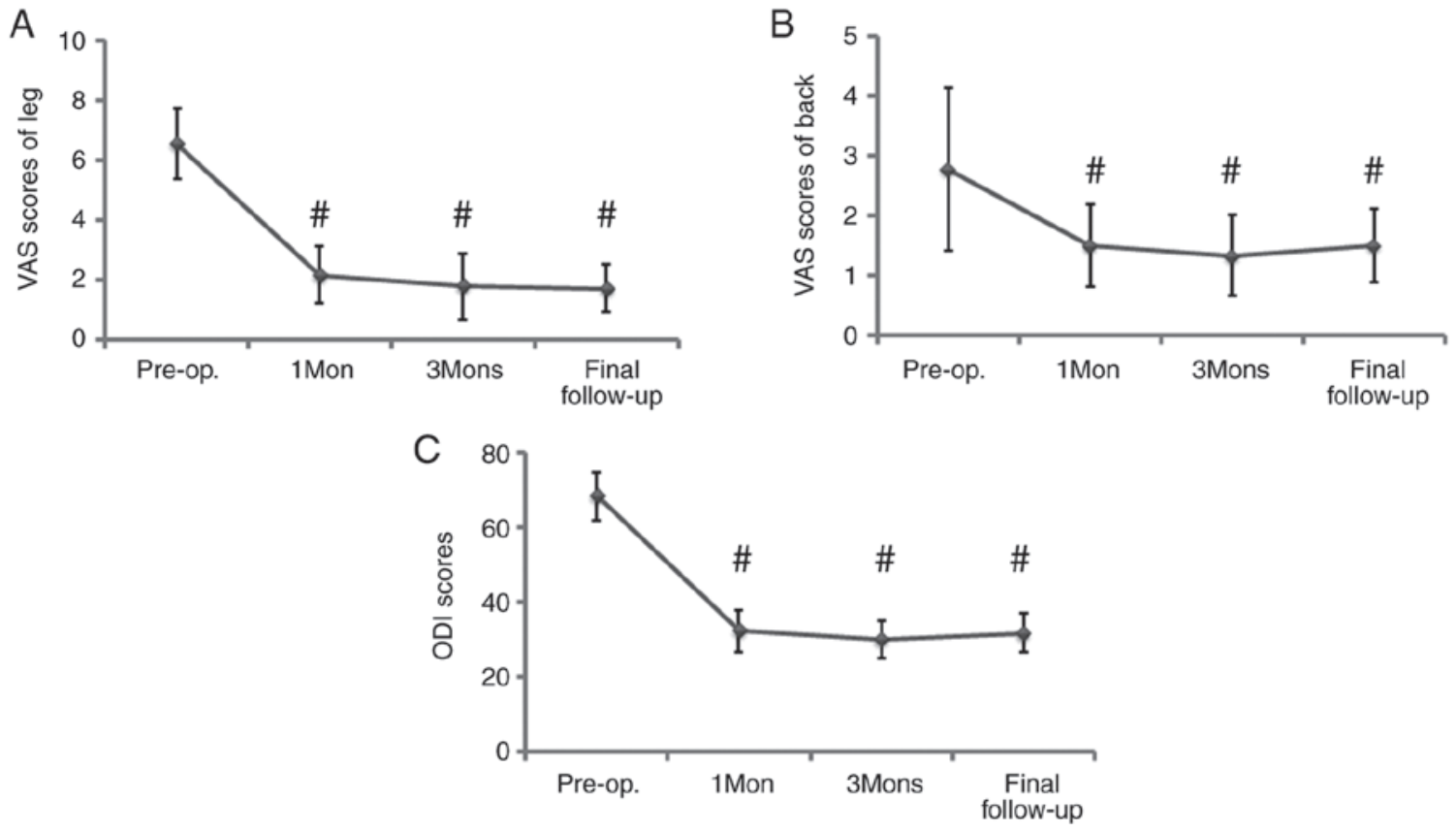

Figure 3. Comparison of (A and B) VAS scores of (A) leg and (B) back, and (C) ODI at different follow-up time-points. " $\mathrm{P}<0.05$ vs. pre-operation group. VAS, visual analogue scale; ODI, Oswestry Disability Index; Mon, month.

was reported in $83.3 \%$ of cases, and only $5.6 \%$ of patients complained of worsening (Fig. 4).

Radiological outcomes. Following surgery, CT and MRI images were evaluated for the assessment of decompression. Fig. 5 represents the case of an 81-year-old male patient exhibiting right leg-dominant symptoms with DLS and spinal stenosis. The pre- and post-surgery images were captured and compared. CT scans and MRI images demonstrated that right foramina stenosis and nerve compression were present (Fig. 5A and C), and following surgery, the removal of the thickened ligaments and the osteophyte of the facet joints pressing on the nerve was evidenced (Fig. 5B and D). As presented in Fig. 6 , the average percent slippage was $18.1 \pm 4.3 \%$ prior to 
Table I. Demographics of the cohort of the present study $(n=18)$.

\begin{tabular}{|c|c|}
\hline Parameter & Value \\
\hline Age (years) & $71.2(66-85)$ \\
\hline Female sex & $10(55.6)$ \\
\hline Duration of symptoms (months) & $5.3(3-12)$ \\
\hline \multicolumn{2}{|l|}{ Levels involved } \\
\hline L4-5 & $12(66.7)$ \\
\hline L5-S1 & $6(33.3)$ \\
\hline \multicolumn{2}{|l|}{ Number of levels operated } \\
\hline One level & $18(100)$ \\
\hline Two level & $0(0)$ \\
\hline \multicolumn{2}{|l|}{ Spondylolisthesis } \\
\hline Meyerding grade I & $13(72.2)$ \\
\hline Meyerding grade II & $5(27.8)$ \\
\hline Body mass index & $24.1(27.1-21.1)$ \\
\hline \multicolumn{2}{|l|}{ Prevalence of comorbidities } \\
\hline Hypertension & $61.1 \%$ \\
\hline Diabetes mellitus & $27.8 \%$ \\
\hline Heart disease & $38.9 \%$ \\
\hline Cerebrovascular infarction & $11.1 \%$ \\
\hline Respiratory diseases & $16.7 \%$ \\
\hline Renal/ureteral disease & $16.7 \%$ \\
\hline Peripheral vascular disease & $11.1 \%$ \\
\hline Follow-up (months) & $27.7(24-33)$ \\
\hline Blood loss (ml) & $12.8(10-25)$ \\
\hline Duration of surgery (min) & $90.6(50-120)$ \\
\hline Hospital stay (days) & $1.4(1-5)$ \\
\hline
\end{tabular}

Values are expressed as the median (range) or $\mathrm{n}(\%)$.

surgery and $18.9 \pm 4.8 \%$ at the final follow-up. No statistically significant differences were observed between the percentage of lumbar spondylolisthesis prior to surgery and at the end of follow-up $(\mathrm{P}>0.05)$.

Complications and recurrence. Complications occurred in 3 patients. A total of 2 small dural tears were detected intra-operatively, which were not repaired at the time of surgery. No permanent neurological sequelae were indicated during the follow-up period of these 2 patients. A single patient with sciatica exhibited sciatica-associated symptoms for three months following surgery. Non-surgical treatment failed and this patient underwent micro-decompression surgery. At the final follow-up, the patient's symptoms of sciatica were relieved. No post-operative major complications, including neuro-vascular injury, cauda equina injury or surgical wound infection were recorded.

\section{Discussion}

The use of percutaneous transforaminal endoscopic techniques is popular in the treatment of patients with lumbar
Table II. Outcomes according to the modified MacNab criteria.

\begin{tabular}{|c|c|c|}
\hline Outcome & Description & $\mathrm{n}(\%)$ \\
\hline Excellent & Complete relief of symptoms & $10(55.6)$ \\
\hline Good & $\begin{array}{l}\text { Marked improvement but occasional } \\
\text { pain }\end{array}$ & $5(27.8)$ \\
\hline Fair & $\begin{array}{l}\text { Improved functional capacity and the } \\
\text { need for pain medications }\end{array}$ & $2(11.1)$ \\
\hline Poor & Unimproved symptoms or worsening & $1(5.6)$ \\
\hline
\end{tabular}

degenerative diseases (10). DLS associated with spinal stenosis is a common clinical condition that significantly contributes to pain and disability, particularly in the elderly (1). However, few studies have assessed the application of transforaminal endoscopic techniques in the treatment of this condition $(18,19)$. The present retrospective indicated that a cohort of elderly patients with DLS experienced symptom relief following PTED surgery. The data of $\geq 2$ years of follow-up demonstrated that transforaminal endoscopic decompression under local anesthesia is a feasible and safe procedure, and this technique may provide an alternative treatment option for a proportion of elderly patients exhibiting DLS with spinal stenosis.

Open surgery with general anesthesia for the lumbar spine remains challenging. Multiple factors, including old age, osteoporosis and other unfavorable factors, may lead to a poor clinical outcome (20). Furthermore, residual axial lower back pain and adjacent-segment degeneration after instrument implantation are commonly indicated in a number of patients (21). The operation procedure for DLS may be divided into simple decompression or decompression combined with fixation (10). The argument for the treatment of DLS is that arthrodesis is used to enhance the stability of the spine and avoid the progression of spondylolisthesis. However, spondylolisthesis has been indicated to rarely progress in adults (22). Recent studies have demonstrated that the additional fusion may not yield obvious clinical improvements over decompression alone, particularly when minimally invasive decompression is used $(1,9)$. The extent of resection of the articular joint or ligamentous elements exhibits a strong influence on the degree of spinal instability (23). Transforaminal endoscopic techniques may preserve the biomechanical structure of the surgical segment and this is improved compared with traditional open surgery. Therefore, transforaminal endoscopic techniques exert a minimal impact on spinal stability.

Microendoscopic discectomy (MED) is a reliable technique for the treatment of DLS (24). During MED, the dorsal ligamentum flavum and part of the lamina are removed to enlarge the volume of the spinal canal. In the transforaminal endoscopic decompression procedure, decompression was achieved via local excision of the ligamentum flavum and ventral superior articular processes. The transforaminal endoscopic procedure may preserve the intact structure of the facet joint capsule. PTED preserves more structures and MED is able to provide more volume for the spinal cord and nerve roots involved. Jang et al (25) retrospectively reviewed 21 patients who underwent MED for spinal stenosis associated with DLS, and the 
Walking distance improvement

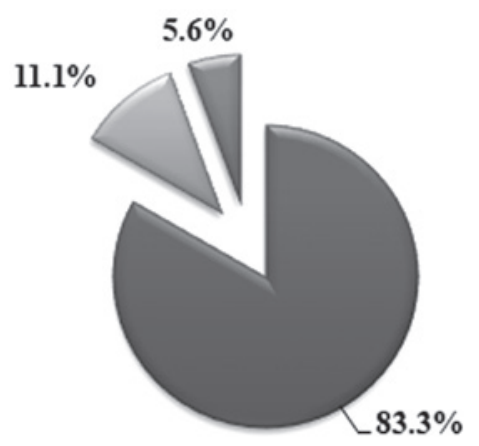

Figure 4. According to the results of walking distance improvement, the estimated walking distance increased and improvement was reported in $83.3 \%$ of cases, and only $5.6 \%$ of patients complained of worsening.
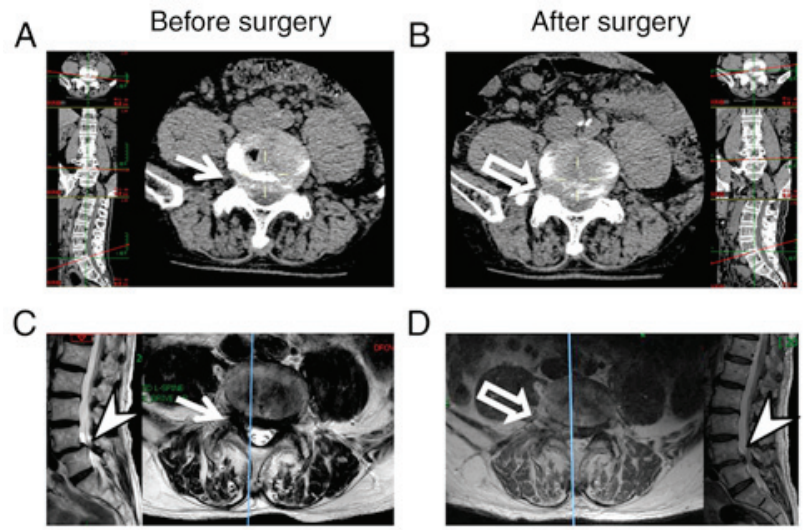

Figure 5. Radiographic results of an 81-year-old male patient who exhibited right leg dominant symptoms with DLS and spinal stenosis that was treated with percutaneous transforaminal endoscopic decompression. (A) CT scans indicating right foramina stenosis and nerve compression prior to the operation (solid arrow). (B) Thorough nerve decompression was indicated in post-operative CT scans (hollow arrow). (C) MRI images showed right foramina stenosis and nerve compression prior to the operation (solid arrow) (D) Complete neurodecompression was shown in T2-weighted MRI images. The removal of the thickened ligaments and the osteophyte of the facet joints pressing on the nerve were indicated (hollow arrow). Comparison of pre- and post-operative T2-weighted MRI images of the sagittal plane demonstrated decompression results (arrowheads). The blue lines indicated the sagittal plane. DLS, degenerative lumbar spondylolisthesis.

ODI score was demonstrated to improve from 59.5 to 26.2 at a minimum follow-up of 3 years. Kelleher et al (26) collected the data of 25 patients with DLS who reported radicular leg pain without severe back pain and obvious spinal instability. In that study, following MED surgery, the ODI score indicated a $48.8 \%$ improvement and $77.8 \%$ of patients were satisfied with this surgical treatment at the final follow-up. In the present study, similar functional improvement was achieved compared with the outcome of MED reported in the the aforementioned studies. The mean decrease in the ODI was 36.5 and the mean decrease of the VAS score of the leg was 5.4 in the present study. Clinical observations demonstrated that the PTE procedure has a number of advantages over MED, including decreased incision length, hospital stay, surgical time, blood loss and muscle damage. Compared with the MED technique, PTED is associated with a more rapid recovery and provides improved clinical outcomes for patients $(11,25)$. However,

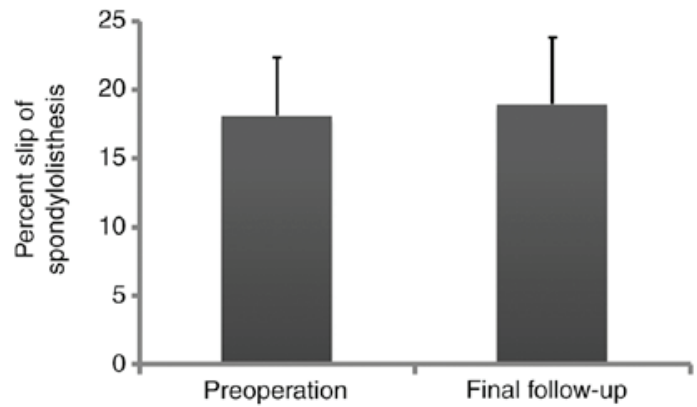

Figure 6. Comparison of the percentage of lumbar spondylolisthesis prior to surgery and at the end of follow-up. No statistically significant differences were observed between the percent slip of spondylolisthesis at the end of follow-up vs. prior to the surgery $(\mathrm{P}>0.05)$.

comparison of the long-term clinical outcomes between PTED and MED is required in future studies.

The transforaminal endoscopic procedure may be used to treat nerve root compression caused by a herniated disc. However, in DLS, the surgeon should ensure that the ventral and dorsal nerves are completely decompressed. In the present study, the dorsal approach decompression procedure was performed using an endoscopic chisel or trephine to remove hypertrophied superior articular processes. The intervertebral foramen was then enlarged under endoscopy to provide a sufficient space for the surgical procedure. Consequently, it was possible to fully resect the target hypertrophied ligament flavum. Due to a small space between the ligamentum flavum and the underlying dural sac, the surgeon should avoid aggressive manipulation of the dura mater or nerve root injury. The ventral approach was always performed after the completion of posterior decompression. To obtain ventral decompression, a working cannula should be retreated to make its transition from the dorsal side to the ventral side safe. The conditions of central stenosis, lateral recess stenosis and combined stenosis were included in the present cohort. The majority of patients with DLS are generally associated with lateral recess stenosis, which is usually caused by a herniated disc, ligamentum flavum hypertrophy and/or joint capsule hypertrophy (27). With the accumulation of experience, percutaneous transforaminal endoscopic techniques may be performed safely, with decreased blood loss and and decreased complication rates of dural tears and wound infection. The majority of dural tears are small and do not require repair during surgery. Revision decompression was required in $5.6 \%$ of patients of the present study, which is similar to the rates in other open surgery studies $(5,28)$.

The transforaminal and interlaminar approach are two common operative procedures used in full endoscopic lumbar spine surgery treatment. The interlaminar approach was initially developed due to the pelvis, particularly at the L5/S1 level, obstructing the established working cannular $(29,30)$. In the present study, all patients with L5/S1-level spondylolisthesis were operated using the transforaminal approach. The interlaminar approach provided an advantage in dorsal decompression. However, extensive exposure of the dural sac may increase the risk of dural sac injury. Furthermore, general anesthesia is required, as local anesthesia is not sufficient during this operation due to dural sac irritation (31). The interlaminar approach has been indicated to be suitable for the 
decompression of central stenosis combined with or without lateral recess stenosis, and the transforaminal procedure is applied to lateral and/or foraminal stenosis. However, these indications may change with the development of surgical instruments and surgeons should use the technique they are familiar with to achieve reliable clinical results. Although patients treated with the transforaminal or interlaminar approach exhibit good short-term clinical outcomes, long-term follow-up is required to determine whether these outcomes deteriorate.

In the present study, all samples were collected from a single research center and the sample size was small, which may reduce the statistical power of the results and limit the scientific value of the conclusions drawn. The statistical power of the present study was calculated for all comparisons. Power analysis validated the results of ODI, VAS of the leg and back pain scores between the pre-operative baseline and post-operative month 1 and 3, as well as the final follow-up with $>98 \%$ certainty. However, the power for comparison of the percentage of lumbar spondylolisthesis between the pre-operative baseline and at the end of the follow-up period was $<70 \%$. The results of the comparison between the percentage of lumbar spondylolisthesis prior to surgery and at the final follow-up may have been partly affected by the small sample size. A study assessing a larger sample size should be performed in the future. The present study was also a retrospective study based on electronic records. Although the information regarding the comorbidities of the patients was provided, data on the severity grading of their pathologies were not obtained. In addition, PTED techniques continue to evolve, and although patients treated with such methods exhibit good short-term clinical outcomes, long-term follow-up is required to determine whether any deterioration occurs.

In conclusion, the present preliminary study demonstrated that PTED alone is a feasible and safe procedure for the treatment of leg-dominant symptoms in elderly patients with lumbar spinal stenosis that is associated with DLS. No significant difference was indicated in the percentage of slippage between the pre-operative stage and the end of the follow-up. The rate of post-surgical revision was low, with $5.6 \%$ of patients requiring a subsequent micro-decompression surgery at a mean follow-up of 27.7 months. PTED under local anesthesia may also be an efficient alternative to conventional open lumbar decompression surgery for the treatment of elderly patients. However, PTED techniques continue to evolve and the efficacy of this technique requires to be further evaluated by a long-term follow-up study.

\section{Acknowledgements}

Not applicable.

\section{Funding}

This study was supported by the National Natural Science Foundation of China (grant nos. 81772292 and 81270027) and by the Medico-Engineering cooperation Fund of Shanghai Jiao Tong University (grant nos. YG2012MS25 and YG2016MS54).

\section{Availability of data and materials}

The datasets used and/or analyzed during the present study are available from the corresponding author on reasonable request.

\section{Authors' contributions}

XFL designed the study and drafted the manuscript. LYJ and XXS recruited the patients and analyzed the data.ZDL and XJS analyzed the data. KW and HXS collected and analyzed the pre-, intra- and post-operative data. All authors reviewed and approved the final manuscript.

\section{Ethics approval and consent to participate}

Ethics approval was obtained from the Ethics Committee of Renji Hospital (Shanghai, China). All patients provided written informed consent.

\section{Patient consent for publication}

All patients provided consent for publication of their data and images.

\section{Competing interests}

The authors declare that they have no competing interests.

\section{References}

1. Scholler K, Alimi M, Cong GT, Christos P and Hartl R: Lumbar spinal stenosis associated with degenerative lumbar spondylolisthesis: A systematic review and meta-analysis of secondary fusion rates following open vs minimally invasive decompression. Neurosurgery 80: 355-367, 2017.

2. Resnick DK, Watters WC III, Sharan A, Mummaneni PV, Dailey AT, Wang JC, Choudhri TF, Eck J, Ghogawala Z, Groff MW, et al: Guideline update for the performance of fusion procedures for degenerative disease of the lumbar spine. Part 9: Lumbar fusion for stenosis with spondylolisthesis. J Neurosurg Spine 21: 54-61, 2014.

3. Austevoll IM, Gjestad R, Brox JI, Solberg TK, Storheim K, Rekeland F, Hermansen E, Indrekvam K and Hellum C: The effectiveness of decompression alone compared with additional fusion for lumbar spinal stenosis with degenerative spondylolisthesis: A pragmatic comparative non-inferiority observational study from the norwegian registry for spine surgery. Eur Spine J 26: 404-413, 2017.

4. Ghogawala Z, Dziura J, Butler WE, Dai F, Terrin N, Magge SN, Coumans JV, Harrington JF, Amin-Hanjani S, Schwartz JS, et al: Laminectomy plus fusion versus laminectomy alone for lumbar spondylolisthesis. N Engl J Med 374: 1424-1434, 2016.

5. Ahmad S, Hamad A, Bhalla A, Turner S, Balain B and Jaffray D: The outcome of decompression alone for lumbar spinal stenosis with degenerative spondylolisthesis. Eur Spine J 26: 414-419, 2017.

6. Inose H, Kato T, Yuasa M, Yamada T, Maehara H, Hirai T, Yoshii T, Kawabata S and Okawa A: Comparison of decompression, decompression plus fusion, and decompression plus stabilization for degenerative spondylolisthesis: A prospective, randomized study. Clin Spine surg 31: E347-E352, 2018.

7. Chang W, Yuwen P, Zhu Y, Wei N, Feng C, Zhang Y and Chen W: Effectiveness of decompression alone versus decompression plus fusion for lumbar spinal stenosis: A systematic review and meta-analysis. Arch Orthop Trauma Surg 137: 637-650, 2017.

8. Alimi M, Hofstetter CP, Pyo SY, Paulo D and Hartl R: Minimally invasive laminectomy for lumbar spinal stenosis in patients with and without preoperative spondylolisthesis: Clinical outcome and reoperation rates. J Neurosurg Spine 22: 339-352, 2015.

9. Minamide A, Yoshida M, Simpson AK, Nakagawa Y, Iwasaki H, Tsutsui S, Takami M, Hashizume H, Yukawa Y and Yamada H: Minimally invasive spinal decompression for degenerative lumbar spondylolisthesis and stenosis maintains stability and may avoid the need for fusion. Bone Joint J 100-B: 499-506, 2018. 
10. Allen RT and Garfin SR: The economics of minimally invasive spine surgery: The value perspective. Spine (Phila Pa 1976) 35 (26 Suppl): S375-S382, 2010.

11. Liu X, Yuan S, Tian Y, Wang L, Gong L, Zheng Y and Li J: Comparison of percutaneous endoscopic transforaminal discectomy, microendoscopic discectomy, and microdiscectomy for symptomatic lumbar disc herniation: Minimum 2-year follow-up results. J Neurosurg Spine 28: 317-325, 2018.

12. Ahn Y, Oh HK, Kim H, Lee SH and Lee HN: Percutaneous endoscopic lumbar foraminotomy: An advanced surgical technique and clinical outcomes. Neurosurgery 75: 124-133; Discussion 132-133, 2014.

13. Shin SH, Bae JS, Lee SH, Keum HJ, Kim HJ and Jang WS: Transforaminal endoscopic decompression for lumbar spinal stenosis: A novel surgical technique and clinical outcomes. World Neurosurg 114: e873-e882, 2018.

14. Li XF, Jin LY, Lv ZD, Su XJ, Wang K, Song XX and Shen HX: Endoscopic ventral decompression for spinal stenosis with degenerative spondylolisthesis by partially removing posterosuperior margin underneath the slipping vertebral body: Technical note and outcome evaluation. World Neurosurg 126: e517-e525, 2019.

15. Downie WW, Leatham PA, Rhind VM, Pickup ME and Wright V: The visual analogue scale in the assessment of grip strength. Ann Rheum Dis 37: 382-384, 1978.

16. Daltroy LH, Cats-Baril WL, Katz JN, Fossel AH and Liang MH: The North American spine society lumbar spine outcome assessment Instrument: Reliability and validity tests. Spine (Phila Pa 1976) 21: 741-749, 1996.

17. Macnab I: Negative disc exploration. An analysis of the causes of nerve-root involvement in sixty-eight patients. J Bone Joint Surg Am 53: 891-903, 1971.

18. Jasper GP, Francisco GM and Telfeian AE: Transforaminal endoscopic discectomy with foraminoplasty for the treatment of spondylolisthesis. Pain Physician 17: E703-E708, 2014.

19. Jasper GP, Francisco GM, Aghion D and Telfeian AE: Technical considerations in transforaminal endoscopic discectomy with foraminoplasty for the treatment of spondylolisthesis: Case report. Clin Neurol Neurosurg 119: 84-87, 2014.

20. Kim CH, Chung CK, Choi Y, Kim MJ, Kim MJ, Shin S, Yang SH, Hwang SH, Kim DH, Park SB and Lee JH: increased proportion of fusion surgery for degenerative lumbar spondylolisthesis and changes in reoperation rate: A nationwide cohort study with a minimum 5-year follow-Up. Spine (Phila Pa 1976) 44: 346-354, 2019.
21. Hsieh MK, Kao FC, Chen WJ, Chen IJ and Wang SF: The influence of spinopelvic parameters on adjacent-segment degeneration after short spinal fusion for degenerative spondylolisthesis. J Neurosurg Spine 29: 407-413, 2018.

22. Winter RB: The natural history of spondylolysis and spondylolisthesis. J Bone Joint Surg Am 67: 823, 1985.

23. Zander T, Rohlmann A, Klockner C and Bergmann G: Influence of graded facetectomy and laminectomy on spinal biomechanics. Eur Spine J 12: 427-434, 2003.

24. Ikuta K, Tono O and Oga M: Clinical outcome of microendoscopic posterior decompression for spinal stenosis associated with degenerative spondylolisthesis-minimum 2-year outcome of 37 patients. Minim Invasive Neurosurg 51: 267-271, 2008.

25. Jang JW, Park JH, Hyun SJ and Rhim SC: Clinical outcomes and radiologic changes after microsurgical bilateral decompression by a unilateral approach in patients with lumbar spinal stenosis and grade I degenerative spondylolisthesis with a minimum 3-year follow-up. Clin Spine Surg 29: 268-271, 2016.

26. Kelleher MO, Timlin M, Persaud O and Rampersaud YR: Success and failure of minimally invasive decompression for focal lumbar spinal stenosis in patients with and without deformity. Spine (Phila Pa 1976) 35: E981-E987, 2010.

27. Dijkerman ML, Overdevest GM, Moojen WA and VleggeertLankamp CLA: Decompression with or without concomitant fusion in lumbar stenosis due to degenerative spondylolisthesis: A systematic review. Eur Spine J 27: 1629-1643, 2018.

28. Chen Z, Zhang L, Dong J, Xie P, Liu B, Wang Q, Chen R, Feng F, Yang B, Shu T, et al: Percutaneous transforaminal endoscopic discectomy compared with microendoscopic discectomy for lumbar disc herniation: 1-year results of an ongoing randomized controlled trial. J Neurosurg Spine 28: 300-310, 2018.

29. Forsth P, Michaelsson K and Sanden B: Does fusion improve the outcome after decompressive surgery for lumbar spinal stenosis? A two-year follow-up study involving 5390 patients. Bone Joint J 95-B: 960-965, 2013.

30. Ruetten S, Komp M and Godolias G: A New full-endoscopic technique for the interlaminar operation of lumbar disc herniations using 6-mm endoscopes: Prospective 2-year results of 331 patients. Minim Invasive Neurosurg 49: 80-87, 2006.

31. Nie H, Zeng J, Song Y, Chen G, Wang X, Li Z, Jiang H and Kong Q: Percutaneous endoscopic lumbar discectomy for L5-S1 disc herniation via an interlaminar approach versus a transforaminal approach: A prospective randomized controlled study with 2-year follow up. Spine (Phila Pa 1976) 41 (Suppl 19): B30-B37, 2016. 